University of Nebraska - Lincoln

DigitalCommons@University of Nebraska - Lincoln

10-1-2005

\title{
Coarse-grained free-energy-functional treatment of quasistatic multiscale processes in heterogeneous materials
}

\author{
H. Zhou \\ University of Nebraska-Lincoln \\ Ruqiang Feng \\ University of Nebraska-Lincoln, rfeng1@unl.edu \\ Dennis J. Diestler \\ University of Nebraska-Lincoln, ddiestler1@unl.edu \\ Xiao Cheng Zeng \\ University of Nebraska-Lincoln, xzeng1@unl.edu
}

Follow this and additional works at: https://digitalcommons.unl.edu/chemzeng

Part of the Chemistry Commons

Zhou, H.; Feng, Ruqiang; Diestler, Dennis J.; and Zeng, Xiao Cheng, "Coarse-grained free-energy-functional treatment of quasistatic multiscale processes in heterogeneous materials" (2005). Xiao Cheng Zeng Publications. 15.

https://digitalcommons.unl.edu/chemzeng/15

This Article is brought to you for free and open access by the Published Research - Department of Chemistry at DigitalCommons@University of Nebraska - Lincoln. It has been accepted for inclusion in Xiao Cheng Zeng Publications by an authorized administrator of DigitalCommons@University of Nebraska - Lincoln. 


\title{
Coarse-grained free-energy-functional treatment of quasistatic multiscale processes in heterogeneous materials
}

\author{
H. Zhou and R. Feng \\ Department of Engineering Mechanics, University of Nebraska-Lincoln, Lincoln, Nebraska 68588 \\ D. J. Diestler \\ Department of Agronomy and Horticulture, University of Nebraska-Lincoln, Lincoln, Nebraska 68583 \\ X. C. Zeng ${ }^{\text {a) }}$ \\ Department of Chemistry, University of Nebraska-Lincoln, Lincoln, Nebraska 68588
}

(Received 19 July 2005; accepted 22 August 2005; published online 27 October 2005)

\begin{abstract}
A new treatment of quasistatic (reversible) multiscale processes in heterogeneous materials at nonzero temperature is presented. The system is coarse grained by means of a finite-element mesh. The coarse-grained free-energy functional (of the positions of the nodes of the mesh) appropriate to the thermodynamic-state variables controlled in the relevant process is minimized. Tests of the new procedure on a Lennard-Jonesium crystal yield thermomechanical properties in good agreement with the "exact" atomistic results. () 2005 American Institute of Physics. [DOI: 10.1063/1.2064607]
\end{abstract}

\section{INTRODUCTION}

Such processes as the propagation of defects in crystalline materials and thin-film lubrication are intrinsically multiscale in that they entail the coupling of highly heterogeneous microscopic regions of the system with homogeneous macroscopic regions. Although the course of evolution of the heterogeneous regions is governed mainly by irregular largeamplitude (diffusive) atomic movements, the response of homogeneous regions, where atoms merely oscillate with small amplitude about their relatively fixed equilibrium positions, cannot be neglected. For realistic systems it is practically impossible to track all atoms by solving the equations of motion. To manage the computational burden and yet achieve a reliable "global" description, one must judiciously merge the atomistic description required for the heterogeneous regions with a coarse-grained description that is adequate for the homogeneous regions. Several computational schemes based on this notion have been proposed since the early 1990s. ${ }^{1-3}$ These have been reviewed exhaustively over the last several years. ${ }^{4-7}$

We confine our attention to quasistatic (reversible) processes. A prominent quasistatic multiscale approach is the quasicontinuum (QC) method, ${ }^{2,4-7}$ which blankets the crystal lattice with a finite-element coarse-graining mesh, thereby eliminating the myriad original atoms in favor of many fewer nodes of the mesh, which act as quasiparticles whose interaction is mediated by the underlying atoms constrained to move in lockstep with the nodes. The coarse-grained potential energy $U_{c}$ is approximated as a sum of contributions from the elements. In the vast homogeneous regions far from defects elements are large compared with the range $r_{c}$ of interaction between atoms. Such a large element is termed "local" because its contribution to $U_{c}$ depends on the con-

${ }^{a)}$ Electronic mail: xczeng@phase2.unl.edu figuration of only its own nodes. As a defect is approached, the elements must gradually become smaller in order to describe the increasing heterogeneity. When the dimension of an element approaches $r_{c}$, it becomes "nonlocal" in that its contribution to $U_{c}$ depends not just on the configuration of its own nodes but also on the configurations of the nodes of neighboring elements within a distance $r_{c}$. The optimum (static) configuration of all nodes at zero temperature ( $T=0 \mathrm{~K}$ ) is found by minimizing $U_{c}$ (as a functional of the nodal configuration) under given boundary conditions. By incrementally varying the boundary conditions so as to mimic a prescribed application of stresses or strains, one can trace the quasistatic evolution of the (metastable) defects.

The restriction on the QC method to $T=0 \mathrm{~K}$ becomes crucial when the thermal energy of an atom $\left(k_{B} T\right.$, where $k_{B}$ is Boltzmann's constant) becomes comparable with its binding energy $\varepsilon$. For example, applying the QC technique to a van der Waals crystal for which $k_{B} T / \varepsilon=0.3$ can lead to an error greater than $100 \%$ in the mean stress (see Table I). Hence, the purpose of this paper is to introduce a new quasistatic multiscale treatment that avoids the zero-temperature restriction on the original QC technique. ${ }^{2}$ In essence we replace the potential-energy functional $U_{c}$ with a free-energy functional $F_{c}$ apropos of the thermodynamic-state variables that are controlled in the process of interest. We report the results of preliminary tests of the new technique on an ideal system, namely, pure face-centered-cubic (fcc) Lennard-Jonesium $(\mathrm{LJ})$ at fixed density $(\rho)$ and temperature $(T)$, whose thermomechanical properties are known or can be readily and accurately computed by independent methods. The relevant free energy that is minimum under these conditions (fixed $\rho, T$ ) is the Helmholtz energy. Thus, the basic idea, which was previously proposed and applied to a one-dimensional model, ${ }^{8}$ is to minimize a Helmholtz-energy functional for the coarsegrained system. 
TABLE I. Mean stress $\tau$ for selected thermodynamic states $(\rho, T)$ of the LJ crystal. Cube $(L=42 a)$ coarse grained in two steps: first partitioned into rectangular prisms by passing sets of planes normal to the $x, y$, and $z$ axes (i.e., planes $x=12 a, x=18 a, x=21 a, x=24 a, x=30 a$, and so on for $y$ and $z$ axes); six tetrahedra inscribed in each prism. "Exact" stress determined by Monte Carlo method.

\begin{tabular}{ccccc}
\hline \hline$\rho$ & $T$ & $\tau_{\text {exact }}$ & $\tau$ & \% error \\
\hline \multirow{2}{*}{1.19} & 0.00 & -9.17 & -9.17 & 0.00 \\
& 0.05 & -9.67 & -9.68 & 0.09 \\
& 0.10 & -10.17 & -10.19 & 0.22 \\
& 0.20 & -11.15 & -11.20 & 0.51 \\
& 0.30 & -12.12 & -12.22 & 0.87 \\
1.11 & 0.00 & -1.00 & -1.00 & 0.00 \\
& 0.05 & -1.49 & -1.50 & 0.82 \\
& 0.10 & -1.95 & -1.99 & 2.13 \\
& 0.20 & -2.85 & -2.98 & 4.54 \\
& 0.30 & -3.75 & -3.97 & 5.82 \\
\hline \hline
\end{tabular}

\section{BENCHMARK SYSTEM}

Figure 1 displays a schematic of the system: a portion of LJ crystal within an imaginary cube of side length $L=n_{c} a$, where $n_{c}$ is the number of fcc unit cells along an edge and $a$ is the cell constant. The total number of atoms in the cube is $N=4 n_{c}^{3}$. The density is related to the cell constant by $\rho=4 / a^{3}$. To avoid edge effects we impose periodic boundary conditions on the faces of the cube. The system is coarse grained by covering it with a finite-element mesh so constructed as to fulfill the periodic boundary conditions.

\section{FREE-ENERGY FUNCTIONAL METHOD}

Assuming that elements are subjected only to homogeneous deformations, we can express the coarse-grained Helmholtz energy approximately as

$$
F_{c}=\sum_{e=1}^{N_{e}} N_{a}^{e} f_{e},
$$

where the summation on $e$ runs over all $N_{e}$ elements, $N_{a}^{e}$ is the number of atoms under element $e$, and $f_{e}$ is the Helmholtz energy per atom. To obtain a computationally practical approximation to $f_{e}$, we invoke the local harmonic approximation, ${ }^{9}$ which yields

$$
f_{e}=u_{e}+3 k_{B} T \ln \left[h\left(\operatorname{det} \boldsymbol{D}_{e}\right)^{1 / 6} / 2 \pi k_{B} T\right] .
$$

In Eq. (2) $u_{e}$, the potential energy per atom of the LJ crystal at $T=0 \mathrm{~K}$, is approximated as a sum of interactions

$$
u_{e}=\frac{1}{2} \sum_{j \neq i} \varphi\left(r_{i j}\right)=\frac{1}{2} \sum_{j \neq i} 4 \varepsilon\left[\left(\sigma / r_{i j}\right)^{12}-\left(\sigma / r_{i j}\right)^{6}\right] \text {, }
$$

where $i$ designates the "representative" atom underlying element $e$ (i.e., the atom nearest the centroid of $e$ ) and the summation on $j$ runs over atoms that lie within a "cutoff" sphere of radius $r_{c}$ centered on $i$. Likewise, in the second term on the right side of Eq. (2), $\boldsymbol{D}_{e}$ is the dynamical matrix associated with the representative atom in the field of its neighbors fixed at their $(T=0 \mathrm{~K})$ equilibrium positions and $h$ is Planck's constant.

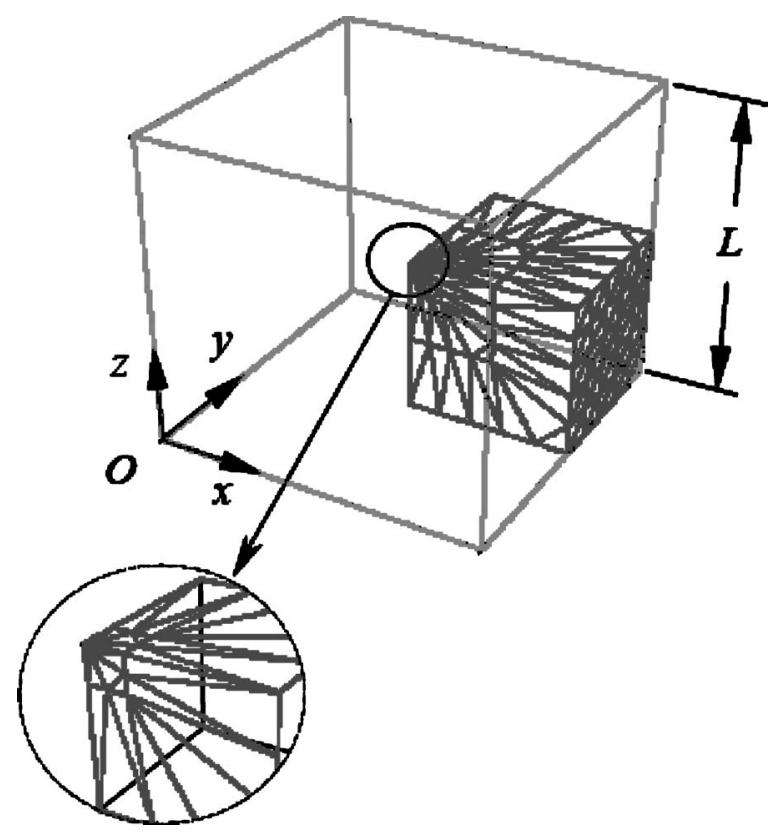

FIG. 1. Schematic of cubic crystal depicting particular coarse-graining mesh. Mesh over whole cube constructed by reflecting mesh in one octant successively through the planes $x=L / 2, y=L / 2$, and $z=L / 2$. Mesh within octant generated by software HYPERMESH from triangular mesh specified on faces of octant.

In case element $e$ is local, the cutoff sphere is contained within $e$ and the representative atom "sees" neighboring atoms underlying only itself. Therefore, $u_{e}$ is equivalent to the potential energy per atom for the infinite crystal deformed at $T=0 \mathrm{~K}$ in the same way (determined by the displacements of the nodes from their reference configuration) as the portion of crystal under $e$. Likewise, $\boldsymbol{D}_{e}$ is equivalent to the dynamical matrix associated with an atom in the infinite crystal so deformed. If $e$ is nonlocal, then the representative atom sees neighbors not only under itself but also under all elements intersected by the cutoff sphere. Since the positions of atoms underlying intersected elements depend on the configurations of the nodes of all those elements, it is clear that evaluation of nonlocal contributions to $F_{c}$ is much more demanding computationally than that of local contributions.

To determine the minimum configuration (i.e., the configuration corresponding to the global minimum of the functional $F_{c}$ at the given fixed $\rho$ and $T$ ), we employ the conjugate gradient method. ${ }^{10}$ The initial configuration of nodes is randomized by assigning each node a random displacement that lies within a small cube of edge $0.15 \sigma$ centered on the reference position of the node. The reference configuration is that of the perfect crystal at $T=0 \mathrm{~K}$. All quantities are given here and elsewhere in the article in dimensionless units based on the parameters of the $\mathrm{LJ}$ interatomic potential [see Eq. (3)]: distance in units of $\sigma$, energy in units of $\varepsilon$, stress in units of $\varepsilon / \sigma^{3}$, and temperature in units of $\varepsilon / k_{B}$. (Note that for argon we have the following MKS units for distance, energy, and stress: $\sigma=3.405 \times 10^{-10} \mathrm{~m} ; \varepsilon=1.654 \times 10^{-21} \mathrm{~J}$; $\varepsilon / \sigma^{3}=0.0419 \mathrm{GPa}$.)

\section{RESULTS AND DISCUSSION}

The thermomechanical property that we focus on is the stress. We consider only thermodynamic states in which the 


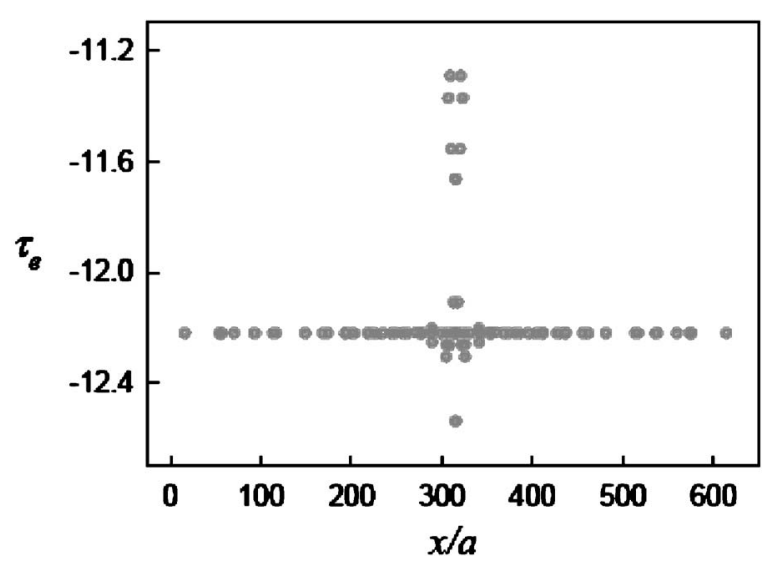

FIG. 2. Coarse-grained mean stress $\left(\tau_{e}\right)$ in elements bordering the midplane $z=L / 2$ vs distance along the $x$ axis. $L=630 a, N=1000188000, N_{e}=4264$, $N_{n}$ (number of nodes) $=1035, \rho=1.19$, and $T=0.3$. Cube coarse grained as depicted in Fig. 1. Data plotted at positions $(x, y)$ of centroids of triangular faces of elements lying in midplane (see Fig. 3 ) and centroids projected onto the $x$ axis.

fcc structure is preserved and compute the global mean stress (i.e., 1/3 of the trace of the stress tensor), which is related to the Helmholtz energy by

$$
\tau=\left(3 L^{2}\right)^{-1}\left(\partial F_{c} / \partial L\right)_{N, T}
$$

Combining Eqs. (1)-(4), we obtain

$$
\tau=L^{-3} \sum_{e=1}^{N_{e}} V_{e} \tau_{e},
$$

where the elemental stress is defined by

$$
\begin{aligned}
\tau_{e}= & N_{a}^{e}\left(6 V_{e}\right)^{-1}\left\{\sum_{j \neq i} r_{i j}\left[d \varphi\left(r_{i j}\right) / d r_{i j}\right]\right. \\
& \left.+k_{B} T \sum_{l} \boldsymbol{r}_{l} \cdot \nabla_{r_{l}}\left[\ln \left(\operatorname{det} \boldsymbol{D}_{e}\right)\right]\right\} .
\end{aligned}
$$

In Eq. (6) $V_{e}$ is the volume of $e$ and the summations on $j$ and $l$ are restricted to atoms within the cutoff sphere. All quantities in Eq. (6) are evaluated at the minimum nodal configuration.

To give the new multiscale treatment a severe test, we coarse grain a large crystal $(L=630 a, N=1000188000)$ with a nonuniform mesh (see Fig. 1), as if there were a highly heterogeneous core. The dimensions of elements vary from $2 a$ at the center of the cube to about $100 a$ at the faces. At the given density of $1.19, a \approx 1.5$. Therefore, since $r_{c}$ $=2.5$, elements in the core are nonlocal. Figure 2 displays a plot of $\tau_{e}$ in elements bordering the midplane $(z=L / 2)$. Except in elements at the core, $\tau_{e}$ is constant over the entire midplane and agrees with the "exact" mean stress $\left(\tau_{\text {exact }}\right.$, see Table I). This result is in harmony with one's expectation that the stress should be uniform throughout the crystal in a state of thermodynamic equilibrium.

The discrepancies in $\tau_{e}$ at the core are traceable to socalled "ghost forces" on the nodes. ${ }^{4,5,11}$ These spurious forces, which lead to false minima in $F_{c}$, are due to asymmetric contributions in regions where elements are nonlocal (e.g., at the core of the cube, in the present instance). Prescriptions to eliminate ghost forces have been suggested. ${ }^{4,5,11}$

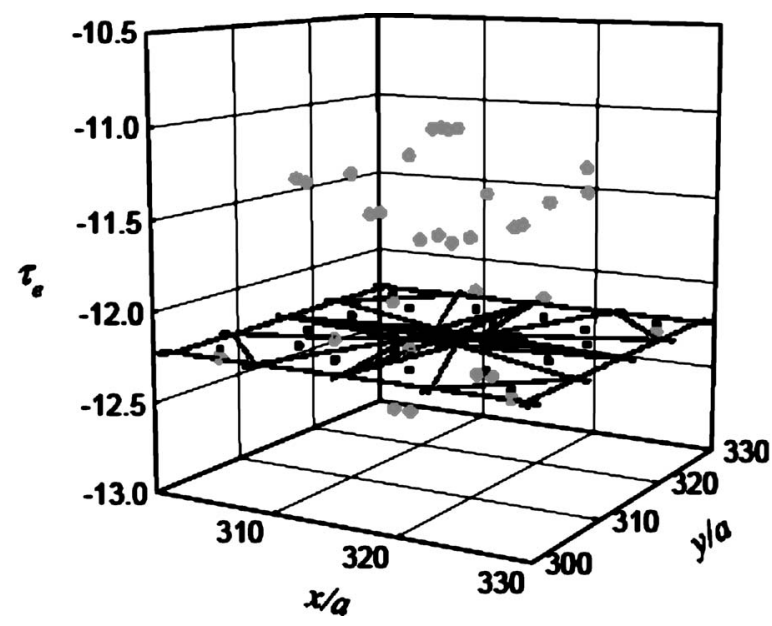

FIG. 3. Perspective plots of coarse-grained mean stress $\left(\tau_{e}\right)$ in elements bordering the midplane $z=L / 2 . \tau_{e}$ based on "all-local" approximation in filled circles; $\tau_{e}$ that accounts for nonlocality of elements in open circles. The plane of triangular mesh lies at the mean value $(-12.22)$.

However, since all these require handling nonlocal elements explicitly, they are very intensive computationally, as mentioned above. Here we attempt to circumvent the problem of ghost forces by a less computationally expensive alternative: we simply treat the nonlocal elements as if they were local. The result is shown in Fig. 3, where we compare this "alllocal" (i.e., all elements are regarded as being local, irrespective of their true status) approximation with the usual procedure which takes account of nonlocality (see Fig. 2). Note that the plots cover only the core region where there are discrepancies. The $\tau_{e}$ based on the all-local approximation (filled circles) is constant across the midplane (indeed, everywhere) and agrees with the global stress $\tau$ as it should.

That such an apparently crude approximation works so well is perhaps surprising. To indicate its limitations we consider a cubic LJ crystal subjected to a sharp, nonuniform static deformation characterized by the displacement field

$$
\begin{aligned}
\boldsymbol{u}(x, y, z)= & 0.1 \sigma \exp \left\{-\left[(x-L / 2)^{2}+(y-L / 2)^{2}\right.\right. \\
& \left.\left.+(z-L / 2)^{2}\right]^{1 / 2} / 3\right\} \boldsymbol{e}_{z}=u_{z} \boldsymbol{e}_{z},
\end{aligned}
$$

where $\boldsymbol{e}_{z}$ is the unit vector. In this case we construct a uniform coarse-graining mesh by subdividing the whole $12 a$ $\times 12 a \times 12 a$ cube into $8^{3}$ smaller $1.5 a \times 1.5 a \times 1.5 a$ cubes and inscribing six tetrahedra in each. The dimension of elements is of the order 2.25, which is less than $r_{c}$, so all elements are actually nonlocal. In Fig. 4 we plot the displacements $\boldsymbol{u}_{z}$ of atoms that lie on a "reference" line parallel with the $x$ axis before the deformation is applied. Also shown are plots of the mean stress at these atoms and in the elements through which the reference line passes. As Fig. 4 indicates, the all-local $\tau_{e}$ is in good agreement with the exact atomic stress, except in the core region $(5.5 a<x<6.5 a)$. On average it does as well as its non-local counterpart. We conclude that treating all elements as local is a reliable approximation, if the deformation gradient is not too large.

To get some information on the range of applicability of the free-energy-functional method, we computed $\tau$ for a selection of thermodynamic states. The results are given in Table I. Also included is the stress obtained by standard 


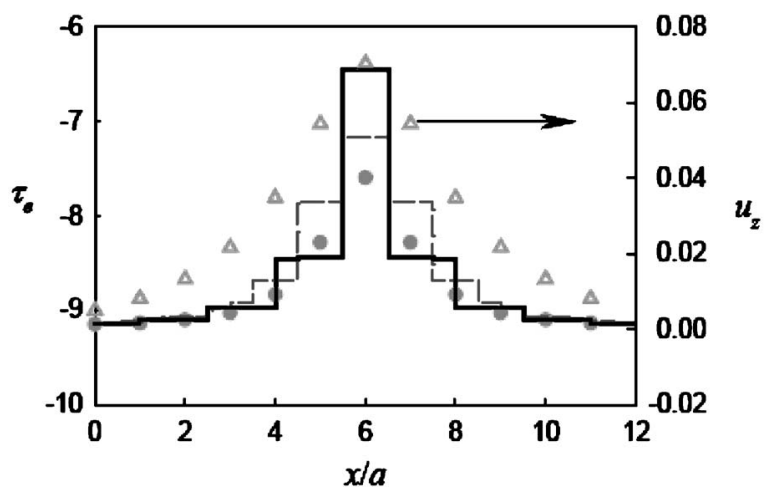

FIG. 4. Coarse-grained mean stress $\left(\tau_{e}\right)$ (left ordinate) and atomic displacement $\boldsymbol{u}_{z}$ (right ordinate) vs distance along "reference" line through cube parallel with $x$ axis, intersecting point $[0,(L-a) / 2,(L-a) / 2]$ (see Fig. 1). $L=12 a, N=6912, N_{e}=3072, N_{n}=729, \rho=1.19$, and $a \approx 1.5$. Displacements of atoms intersected by reference line (before deformation) in triangles (with respect to the right axis); $\tau_{e}$ based on all-local expression [Eq. (6)] in solid lines; $\tau_{e}$ that accounts for nonlocality of elements in dashed lines; "exact" (atomic) stress at intersected atoms in circles.

Metropolis Monte Carlo simulation, ${ }^{12}$ which treats all atoms explicitly. For purposes of comparison, we take the atomistic values to be exact. Two major trends are noted in Table I. First, at fixed $\rho$ the accuracy of the method decreases with increasing $T$. This is due to the harmonic approximation's becoming less reliable as the "real" underlying atoms make thermal excursions into anharmonic regions. Second, at fixed $T$ the error in the stress increases with decreasing $\rho$. At the lower density the effective potential energy binding an atom to its (equilibrium) site is "softer" and more anharmonic than at the higher density. We conclude that the new quasistatic multiscale technique is valid over a considerable range of thermodynamic states where the local harmonic approximation is expected to hold.

Finally, we remark that the ultimate goal of this research is to develop a hybrid atomistic and coarse-grained modeling technique for computing the thermomechanical response of systems that consist of both highly heterogeneous microscopic regions and relatively homogeneous macroscopic regions. Such an approach requires a good coupling of atomistic simulations (for the heterogeneous microscopic regions) with the coarse graining (for the homogeneous macroscopic regions). The progress made in the current work is an important step in this direction. Work to incorporate the two types of simulations into a unified multiscale modeling is under way.

\section{ACKNOWLEDGMENTS}

This research was supported by the National Science Foundation (Grant Nos. DMII/NIRT-0210850 and CHE/ITR0427746), the U.S. DOE (DE-FG02-04ER46164), the U.S. Army Research Office (Grant No. DAAD19-00-1-0161), the Nebraska Research Initiative, and the Research Computing Facility at the University of Nebraska-Lincoln.

${ }^{1}$ S. Kohlhoff, P. Gumbsch, and H. F. Fischmeister, Philos. Mag. A 64, 851 (1991).

${ }^{2}$ E. B. Tadmor, M. Ortiz, and R. Phillips, Philos. Mag. A 66, 1529 (1996). ${ }^{3}$ F. F. Abraham, J. Q. Broughton, N. Bernstein, and E. Kaxiris, Europhys. Lett. 44, 783 (1998).

${ }^{4}$ R. E. Miller and E. B. Tadmor, J. Comput.-Aided Mater. Des. 9, 203 (2002).

${ }^{5}$ W. A. Curtin and R. E. Miller, Modell. Simul. Mater. Sci. Eng. 11, R33 (2003).

${ }^{6}$ R. E. Miller, Int. J. Multiscale Comp. Eng. 1, 57 (2003).

${ }^{7}$ H. S. Park and W. K. Liu, Comput. Methods Appl. Mech. Eng. 193, 1733 (2004).

${ }^{8}$ D. J. Diestler, Phys. Rev. B 66, 184104 (2002).

${ }^{9}$ J. Rickman and R. LeSar, Annu. Rev. Mater. Res. 32, 195 (2002).

${ }^{10}$ W. H. Press, S. A. Teukolsky, W. T. Vetterling, and B. P. Flannery, Numerical Recipes in Fortran 77: The Art of Scientific Computing, 2nd ed. (Cambridge University Press, Cambridge, 1996), Chap. 10.

${ }^{11}$ V. B. Shenoy, R. Miller, E. B. Tadmor, D. Rodney, R. Phillips, and M. Ortiz, J. Mech. Phys. Solids 47, 611 (1999).

${ }^{12}$ M. P. Allen and D. J. Tildesley, Computer Simulation of Liquids (Clarendon, Oxford, 1987), Chap. 4. 